\title{
Prediction of Ambient Dose Equivalent Rates for 30 Years after the Fukushima Accident and its Technological Development
}

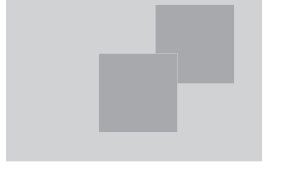

\author{
Japan Atomic Energy Agency, Sakae Kinase
}

\begin{abstract}
The Japan Atomic Energy Agency (JAEA) has been developing a model designed to predict the distribution of ambient dose equivalent rates within $80 \mathrm{~km}$ of the Fukushima Daiichi Nuclear Power Plant (hereinafter referred to as the "Fukushima Daiichi NPP"). A vast amount of measurement data on ambient dose equivalent rates was used to predict changes in the distribution of such rates over a period of 30 years following the Fukushima Daiichi Nuclear Accident by deriving model parameters according to the respective local characteristics inside and outside the evacuation zones. Both uncertainty analysis and validation of this model were conducted. This commentary characterizes the prediction model for the distribution of ambient dose equivalent rates and its parameters. It also presents ambient dose equivalent rate forecast maps that have been generated using this model.
\end{abstract}

KEYWORDS: Ambient dose equivalent rate, prediction model, ecological halflife, cesium, vehicle-borne survey, land use, map, Fukushima

\section{Introduction}

The Fukushima Daiichi Nuclear Accident has compelled us to establish a method for conducting long-term impact assessments to predict the distribution of radioactive cesium deposited in the environment and associated changes in ambient dose equivalent rates. This method is necessary for the provision of basic information that allows residents to keep track of radiation levels, for the selection of appropriate protective measures (including decontamination), and for the reassignment of evacuation zones. Although the deposited radioactive cesium should be identified as a source of radiation for the protection of the public, it is difficult to choose appropriate protective measures due to a lack of sufficient information concerning current and predicted Cs depositions. The experience gained prior to the Fukushima Daiichi Nuclear Accident proved that temporal changes in the radioactive cesium deposited on the ground surface depends on not only radioactive decay, but also weathering effect i.e., impact from changes in the natural environment, such as wind and rain. Making highly reliable predictions is difficult because the radiation levels thus vary in accordance with the natural circumstances of each location and time.

DOI : 10.15669/fukushimainsights.Vol.2.89

(C) 2021 Atomic Energy Society of Japan. All rights reserved.

Originally published in Journal of the Atomic Energy Society of Japan (ISSN 1882-2606), Vol. 58, No. 6, p. 362-366 (2016)

in Japanese. (Japanese version accepted: April 11, 2016) 
The JAEA has been developing a distribution prediction model for ambient dose equivalent rates under a project commissioned initially by the Ministry of Education, Culture, Sports, Science and Technology (MEXT) in FY2012 and subsequently by the Nuclear Regulation Authority (NRA) from FY2013 onwards ${ }^{1-3)}$. In this model, measurement data on ambient dose equivalent rates within $80 \mathrm{~km}$ of the Fukushima Daiichi NPP and data related to land use classification are used for solving an initial value problem. The solutions change from the initial conditions over time until a final solution is produced to predict future distributions. In other words, the model relies on a statistical induction method derived from generalized empirical rules to describe phenomena based on a vast amount of data obtained from extensive investigation of changes in ambient dose equivalent rates according to land use classification. This commentary characterizes the model developed so far for predicting the distribution of and changes in ambient dose equivalent rates. It also presents forecast maps of ambient dose equivalent rates within $80 \mathrm{~km}$ of the Fukushima Daiichi NPP that have been generated using this model to aid the further restoration efforts to be made in Fukushima.

\section{Distribution Prediction Model for Ambient Dose Equivalent Rates}

This model was developed based on a vast amount of measurement data on ambient dose equivalent rates obtained from sources such as a vehicle-borne survey conducted after the Fukushima Daiichi Nuclear Accident. It is aimed at aiding the forecasting of ambient dose equivalent rates from the radioactive cesium deposited extensively within $80 \mathrm{~km}$ of the Fukushima Daiichi NPP. This two-compartment model applies ecological half-lives as parameters derived for the various types of land use within $80 \mathrm{~km}$ of the plant. Here, an ecological half-life is the time required for the ambient dose equivalent rate in the environment to halve due to weathering, human activity, and various other factors other than the decay of radioactive cesium.

\section{Model Equation}

The two-compartment distribution prediction model for which radioactive cesium $\left({ }^{134} \mathrm{Cs}\right.$ and ${ }^{137} \mathrm{Cs}$ ) is the source of the ambient dose equivalent rates is expressed in Equation (1) below.

$$
\begin{gathered}
D(t)=\left(D_{0}-D_{B G}\right)\left\{f_{\text {fast }} \exp \left(\frac{-\ln 2}{T_{\text {fast }}} \cdot t\right)+\left(1-f_{\text {fast }}\right) \exp \left(\frac{-\ln 2}{T_{\text {slow }}} \cdot t\right)\right\} \\
\cdot \frac{k \exp \left(-\lambda_{134} t\right)+\exp \left(-\lambda_{137} t\right)}{k+1}+D_{B G}
\end{gathered}
$$

In this equation, $D(t)$ is the ambient dose equivalent rate at the elapsed time of $t, D_{B G}$ is the average background ambient dose equivalent rate of $0.05 \mu \mathrm{Sv} / \mathrm{h}, T_{\text {slow }}$ is the ecological half-life of 92 years for the slow-decaying component, $k$ is the initial ratio of 2.7 for the ambient dose equivalent rate of ${ }^{134} \mathrm{Cs}$ to that of ${ }^{137} \mathrm{Cs}$ (with the same concentration), $\lambda_{134}$ is the decay constant for ${ }^{134} \mathrm{Cs}$, and $\lambda_{137}$ is the decay constant for ${ }^{137} \mathrm{Cs}$. In Equation (1), the initial ambient dose equivalent rate $D_{0}$ is assigned after data from the vehicle-borne survey and other measurements has been converted into an ambient dose equivalent rate within the habitation zones. The values for both $T_{\text {fast }}$, which is the ecological half-life of the fast-decaying component, 
and $f_{\text {fast }}$, which is a proportion of the fast-decaying component, are derived and assigned based on measurement data obtained from the vehicle-borne survey according to the classification of land use (the cumulative frequency distribution is calculated).

Equation (1) represents the model based on statistical induction. Both $T_{\text {fast }}$ and $T_{\text {slow }}$ depend on the conditions of the deposited radioactive cesium and the types of deposition surfaces. It is difficult to associate these elements quantitatively with the migration mechanism for radioactive cesium in the environment. $T_{\text {fast }}$ is presumably influenced by the shielding effect of the soil as the radioactive cesium migrates deeper into the soil as well as by the reduction in radioactive cesium due to weathering, decontamination, and other forms of human activity. $T_{\text {slow }}$ is most likely influenced by the partial retention of radioactive cesium by moisture on the deposition surface as well as by the redistribution of radioactive cesium by resuspension or human activity. $T_{\text {fast }}$ and $T_{\text {slow }}$ can arguably be considered similar to a distribution phase and an elimination phase, respectively.

\section{Measurement Data on Dose Rates and Model Parameters}

\section{(1) Examination of ambient dose equivalent rate measurement data}

The ambient dose equivalent rates measured since the accident occurred can be roughly divided into data obtained from continuous measurements at monitoring posts and other fixed positions (continuous-time data) and data obtained from irregular measurements taken using mobile instruments during vehicle-borne surveys or the like (discrete-time data). When applied in the distribution prediction model, these two types of data have both advantages and disadvantages in relation to reflecting the characteristics of the ambient dose equivalent rate distributions in terms of time and the relevant location. On the one hand, continuous-time data provides information frequently enough to allow changes in ambient dose equivalent rates in the same spot to be analyzed over time. However, these measurements are taken in a limited number of locations so they are unable to represent an extensive area. On the other hand, discrete-time data provides sufficient information for the characteristics of the ambient dose equivalent rates to be analyzed across an extensive area. However, measurements taken several times in the immediate aftermath of the accident are not sufficient to identify trends over time. For this reason, ecological half-lives were derived as one of the model parameters for developing a distribution prediction model for ambient dose equivalent rates in FY2012. The calculations were based on the results of measurements taken within $20 \mathrm{~km}$ of the plant by TEPCO and an emergency monitoring survey of environmental radiation by Fukushima Prefecture (continuous-time data), as well as the results of vehicle-borne surveys, airborne monitoring, and the like (discrete-time data). With about five years having passed since the accident occurred, sufficient data has been collected from extensive and frequent vehicle-borne surveys and other measurements of the ambient dose equivalent rates within the habitation zones. Such data has made it possible for us to derive and assign the appropriate model parameters for the habitation zones of local residents. Table 1 presents the dates of the vehicle-borne surveys conducted and the number of data items used to derive and assign the model parameters. The eight vehicle-borne surveys commissioned by MEXT and the NRA were conducted within $80 \mathrm{~km}$ of the plant (Surveys 1-8). Other vehicle surveys were conducted inside the evacuation zones in line with a comprehensive monitoring plan (approved at the Monitoring Coordination Meeting). 
Table 1 Vehicle-borne survey data

\begin{tabular}{ll}
\hline Measurement date & No. of data items \\
\hline Survey 1: Jun. 4-13, 2011 & 42,090 \\
Survey 2: Dec. 5-28, 2011 & 33,887 \\
Survey 3: Mar. 13-30, 2012 & 59,037 \\
Survey 4: Aug. 20-Oct. 12, 2012 & 126,249 \\
Survey 5: Nov. 5-Dec. 12, 2012 & 87,991 \\
Survey 6: Jun. 12-Aug. 8, 2013 & 128,270 \\
Survey 7: Nov. 5-Dec. 12, 2013 & 115,985 \\
Survey 8: Jun. 23-Aug. 8, 2014 & 104,630 \\
------------------- \\
conducted in line with the & \\
Trip 1: Aug. 2-30, 2011 & 97,512 \\
Trip 2: Aug. 31-Oct. 9, 2011 & 114,971 \\
Trip 3: Oct. 1-Nov. 4, 2011 & 119,660 \\
Trip 4: Nov. 5-Dec. 12, 2011 & 134,376 \\
Trip 5: Dec. 14, 2011-Jan. 30, 2012 & 135,724 \\
Trip 6: Feb. 4-Mar. 10, 2012 & 132,692 \\
Trip 7: Mar. 12-Apr. 16, 2012 & 144,644 \\
Trip 8: May 16-Jun. 18, 2012 & 145,295 \\
Trip 9: Jul. 4-Aug. 6, 2012 & 148,560 \\
Trip 10: Sep. 4-Oct. 18, 2012 & 153,136 \\
Trip 11: Oct. 31-Dec. 7, 2012 & 152,960 \\
Trip 12: Jan. 9-Feb. 18, 2013 & 142,435 \\
Trip 13: Mar. 18-Apr. 19, 2013 & 153,242 \\
Trip 14: Jun. 4-Jul. 8, 2013 & 154,560 \\
Trip 15: Aug. 27-Oct. 8, 2013 & 155,963 \\
Trip 16: Nov. 6-Dec. 3, 2013 & 154,696 \\
Trip 17: Mar. 18-Apr. 22, 2014 & 158,506 \\
\hline
\end{tabular}

\section{(2) Mesh size within $80 \mathrm{~km}$ of the Fukushima Daiichi NPP}

Ideally, the mesh size of the model for predicting the distribution of ambient dose equivalent rates within $80 \mathrm{~km}$ of the plant should be the same as the zoning of data obtained from the measurements taken so far in order to ensure the convenience and reliability of the predictions. Given that ${ }^{137} \mathrm{Cs}$ has an approximate mean free path of $108 \mathrm{~m}$ in the air (i.e., the average distance a gamma ray travels before colliding with a molecule in the air), a mesh size of about $100 \mathrm{~m}$ was chosen for within $80 \mathrm{~km}$ of the plant. This size corresponds to one-tenth mesh of the third regional compartment adopted for vehicle-borne surveys.

\section{(3) Assignment of initial ambient dose equivalent rates}

In the distribution prediction model for ambient dose equivalent rates, any measurement data obtained from a vehicle-borne survey was applied as initial values as long as such data was available for all meshes within $80 \mathrm{~km}$ of the plant. Otherwise, the measurement data obtained from airborne monitoring was converted into equivalent measurement data by taking into consideration the correlations between data from vehicle-borne surveys and airborne monitoring. In either case, the assigned values were modified according to their proportion in relation to survey data from on-foot surveys to cover the habitation zones of local residents. In the evacuation zones (consisting of difficult-to-return zones, restricted residence zones, and evacuation order cancellation preparation zones), the model mainly applied the detailed monitoring results obtained from vehicle-borne surveys conducted in line with the comprehensive monitoring plan. 


\section{(4) Derivation and assignment of model parameters}

In this model, the ecological half-lives of the slow-decaying component and the range of changes were adopted from values provided by the Atomic Energy Society of Japan and the Nuclear Regulatory Commission of the United States (median value: 92 years; range: 45 to 135 years) with respect to the Level 3 PRA. The ecological half-lives of the fast-decaying component and the range of changes were adopted from values derived by applying the least squares fitting method to the ambient dose equivalent rate measurement data obtained from vehicle-borne surveys. To ensure the reliability and rationality of each ecological half-life for the fast-decaying component in the evacuation zones, two types of half-lives were derived according to the land use classifications by the Advanced Land Observing Satellite (ALOS), which were for deciduous, evergreen and other types of forests as well as other areas. Beyond the evacuation zones, the half-lives were derived by conducting statistical analysis according to the ALOS land use classifications.

Table 2 presents the results of an analysis of the ecological half-lives of the fast-decaying component. The table demonstrates that these ecological half-lives in meshes classified as deciduous or evergreen forests are longer than meshes with other land uses according to the classifications used by the ALOS. In contrast, the latest analysis suggests that the half-lives do not differ significantly for different land uses according to the ALOS classifications. Instead, the difference is notable between inside and outside of the evacuation zones (the ecological half-lives of the fast-decaying component tend to be longer inside evacuation zones with less human activity compared to outside of these evacuation zones). Within a confidence interval of $90 \%$, the statistical distribution of the ecological half-lives of the fast-decaying component could be regarded as a lognormal distribution.

The proportions of the fast-decaying component were derived for both inside and outside of the evacuation zones based on detailed monitoring data obtained from vehicle-borne surveys conducted in line with the comprehensive monitoring plan and ambient dose equivalent rate measurement data obtained from the first eight rounds of vehicle-borne surveys (Surveys 1-8). More specifically, the proportions were derived by applying a non-linear least

Table 2 Ecological half-lives of the fast-decaying component

\begin{tabular}{lccc}
\hline \multicolumn{1}{c}{$\begin{array}{c}\text { ALOS land use } \\
\text { classification }\end{array}$} & \multicolumn{2}{c}{ Half-life of fast-decaying component (year) } \\
5th percentile & Median value & 95th percentile \\
\hline Outside evacuation zones & 0.25 & 0.56 & 1.2 \\
Water areas & 0.35 & 0.60 & 1.7 \\
Urban areas & 0.32 & 0.55 & 1.5 \\
Paddies & 0.32 & 0.63 & 1.9 \\
Dry fields & 0.29 & 0.58 & 2.2 \\
Grass fields & 0.29 & 0.66 & 2.7 \\
Deciduous forests & 0.28 & 0.94 & 5.7 \\
Evergreen forests & 0.31 & 0.62 & 1.6 \\
Bare land & & & \\
\hline Inside evacuation zones & 0.29 & 0.68 & 3.1 \\
Forests & 0.32 & 0.60 & 1.7 \\
Non-forest areas & &
\end{tabular}


squares fitting method with the initial ambient dose equivalent rates and the proportions of the fast-decaying component as the two parameters while assuming a constant ecological half-life for the fast-decaying component. The obtained proportions of the fast-decaying component were classified into forests (deciduous and evergreen) and areas under other types of land use according to the ALOS classifications in evacuation zones comprising the following: difficult-to-return zones, restricted residence zones, and evacuation order cancellation preparation zones. The proportions for outside of the evacuation zones were classified by land use according to the ALOS classifications. Subsequently, their median values and ranges were derived.

Table 3 presents the proportions of the fast-decaying component. Outside the evacuation zones, these proportions are notably different according to land use based on the ALOS classifications. In particular, the proportions in meshes classified as evergreen forests are less than those in meshes classified as urban or other land use areas. A comparison among the difficult-to-return zones, restricted residence zones, and evacuation order cancellation preparation zones that commonly comprise the evacuation zones demonstrates greater proportions and variance of the fast-decaying component in evacuation order cancellation preparation zones with relatively high levels of human activity. Within a confidence interval of $90 \%$, the statistical distribution of all proportions of the fast-decaying component could be regarded as a normal distribution.

Table 3 Proportions of the fast-decaying component

\begin{tabular}{lccc}
\hline \multirow{2}{*}{ ALOS land use classification and attributes } & \multicolumn{2}{c}{ Proportion of fast-decaying component (-) } \\
& 5th percentile & Median value & 95th percentile \\
\hline Outside evacuation zones & & & \\
Water areas & 0.53 & 0.76 & 0.89 \\
Urban areas & 0.52 & 0.77 & 0.93 \\
Paddies & 0.53 & 0.75 & 0.93 \\
Dry fields & 0.48 & 0.71 & 0.89 \\
Grass fields & 0.47 & 0.72 & 0.92 \\
Deciduous forests & 0.41 & 0.68 & 0.88 \\
Evergreen forests & 0.26 & 0.62 & 0.86 \\
Bare land & 0.51 & 0.73 & 0.90 \\
\hline $\begin{array}{l}\text { Inside evacuation zones } \\
\text { Forests in difficult-to-return zones }\end{array}$ & 0.32 & 0.45 & 0.55 \\
Non-forest areas in difficult-to-return zones & 0.33 & 0.47 & 0.57 \\
Forests in restricted residence zones & 0.37 & 0.48 & 0.61 \\
Non-forest areas in restricted residence zones & 0.39 & 0.49 & 0.65 \\
$\begin{array}{l}\text { Forests in evacuation order cancellation } \\
\text { preparation zones }\end{array}$ & 0.37 & 0.51 & 0.67 \\
$\begin{array}{l}\text { Non-forest areas in evacuation order } \\
\text { cancellation preparation zones }\end{array}$ & 0.29 & 0.51 & 0.69 \\
\hline
\end{tabular}




\section{Uncertainty Analysis and Validation of the Model}

\section{(1) Uncertainty analysis of the model}

The prediction model was employed to forecast the distribution of ambient dose equivalent rates for up to 30 years following the Fukushima Daiichi Nuclear Accident both inside and outside of the evacuation zones. In the uncertainty analysis of the model, changes in the ambient dose equivalent rates were estimated using the Monte Carlo method, which considers the statistical distributions of the model's three parameters, as shown in Equation (1); in other words, the proportions of the fast-decaying component, the ecological half-lives of the fast-decaying component, and the ecological half-lives of the slow-decaying component. A uniform distribution was assumed for the statistical distribution of the ecological half-lives of the slow-decaying component based on the findings of earlier studies.

Figures 1(a) to (c) present examples of time changes in the ambient dose equivalent rates estimated using the prediction model. Figure 1(a) presents forecasts for a mesh from an urban area outside of the evacuation zones according to the ALOS land use classifications. Figure 1(b) presents forecasts for a mesh in an area not classified as a deciduous or evergreen forest in a difficult-to-return zone according to the ALOS land use classifications, while Figure 1(c) presents that for the same type of area in an evacuation order cancellation preparation zone. These figures were commonly normalized using the initial ambient dose equivalent rates, excluding the background ambient dose equivalent rates. These figures suggest that the ambient dose equivalent rates predicted by the model attenuate faster compared to scenarios in which only the decay of radioactive cesium is considered and that the attenuation is slower with less human activity. They also plot the relative values of the ambient dose equivalent rates after normalization, as well as the relative values (with each measured value being divided by the corresponding initial ambient dose equivalent rate after subtraction of the background ambient dose equivalent rate) of the measurement data for the ambient dose equivalent rates from the first eight rounds of the vehicle-borne surveys (Surveys 1-8) and up to Trip 17 of the vehicle-borne surveys conducted in line with the comprehensive monitoring plan. The relative values (median values) for the estimated ambient dose equivalent rate agree well with the ambient dose equivalent rates measured in the vehicle-borne surveys, most of which were within the estimated change ranges while taking into consideration uncertainties in relation to the model parameters.

\section{(2) Validation of the model}

The distribution prediction model for ambient dose equivalent rates based on the measurement data obtained from the seventh vehicle-borne survey was validated by comparing the estimate results with the ambient dose equivalent rates measured in the eighth vehicle-borne survey conducted outside of the evacuation zones. The results are presented in Figure 2. The estimated ambient dose equivalent rates and measurements agree well within a factor of 2 for $0.1 \mu \mathrm{Sv} / \mathrm{h}$ and greater, and their agreement improves as the ambient dose equivalent rate grows larger. The discrete trends of the distribution of the ambient dose equivalent rates within the range of around 0.1 to $0.2 \mu \mathrm{Sv} / \mathrm{h}$ is ascribable to the effective digits of the reference measurement data used for the estimation. 


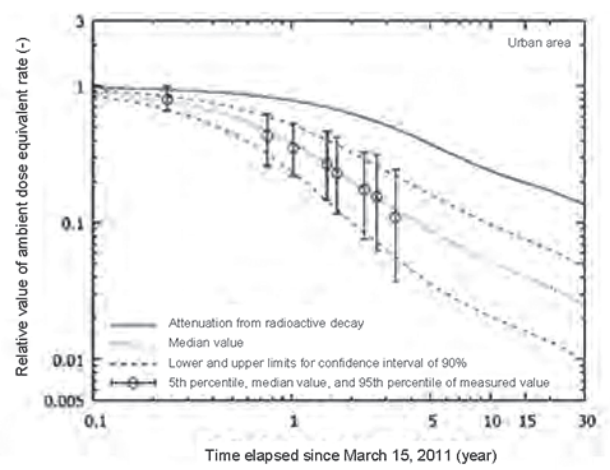

(a) Outside evacuation zones (urban area)

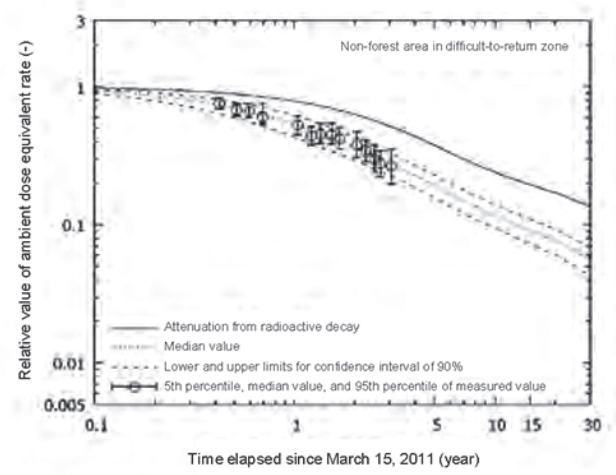

(b) Inside evacuation zones (non-forest area in difficult-to-return zone)

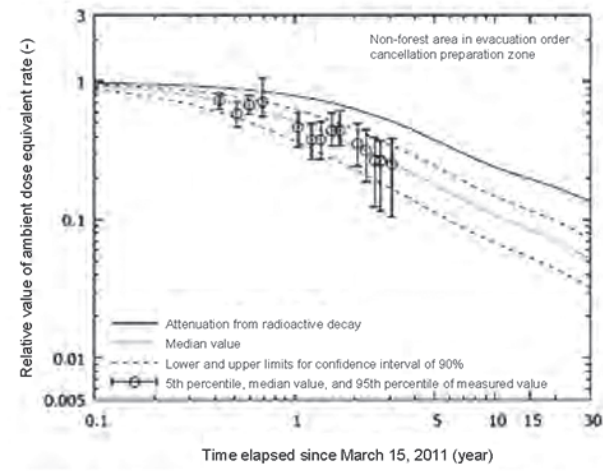

(c) Inside evacuation zones (non-forest area in evacuation order cancellation preparation zone)

Figure 1 Changes in relative values of ambient dose equivalent rates over time 


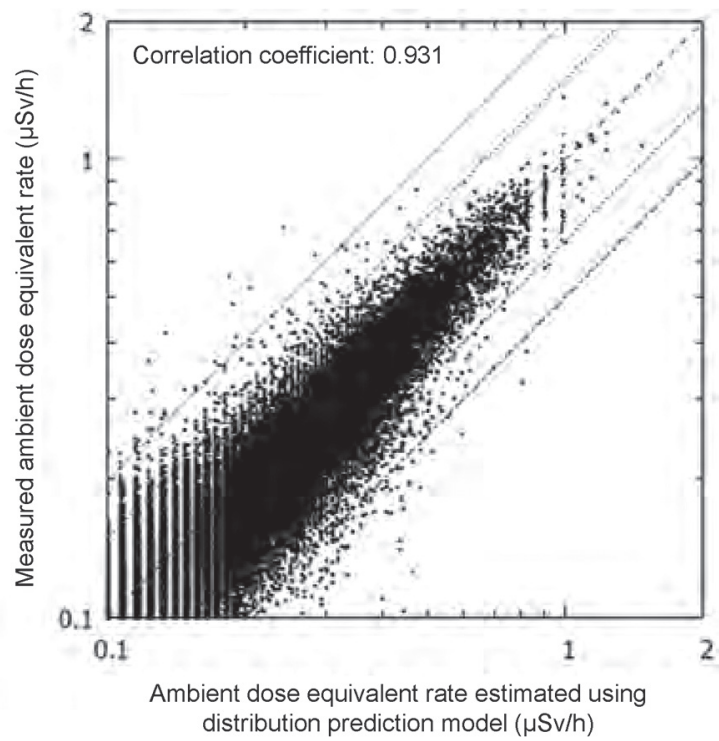

Figure 2 Comparison between ambient dose equivalent rates estimated using the distribution prediction model (based on the seventh vehicle-borne survey) and measurements taken in the eighth vehicle-borne survey

\section{Forecast Maps of Ambient Dose Equivalent Rates}

The physical half-life of ${ }^{137} \mathrm{Cs}$ is around 30 years. With this in mind, forecast maps of the ambient dose equivalent rates were generated for the range within $80 \mathrm{~km}$ of the plant after $5,10,15$, and 30 years after the nuclear accident. The initial ambient dose equivalent rates were adopted from the measurement data obtained from the eighth vehicle-borne survey, Trip 17 of the on-vehicle survey conducted in line with the comprehensive monitoring plan, and the eighth airborne monitoring (as of November 19, 2013). The predictions were conducted in meshes for ambient dose equivalent rates (including the background ambient dose equivalent rate) of over $0.20 \mu \mathrm{Sv} / \mathrm{h}$ by taking into account uncertainties concerning data derived using the ecological half-lives and other model parameters for the distribution prediction model. The forecast was visualized in gray scale according to the estimated levels. Meshes for ambient dose equivalent rates of less than $0.20 \mu \mathrm{Sv} / \mathrm{h}$ are expressed using the same brightness as the level corresponding to $0.20 \mu \mathrm{Sv} / \mathrm{h}$. Meshes without any measurement data from airborne monitoring and vehicle-borne surveys were left blank. Examples include the waters of Lake Inawashiro and the area located within $3 \mathrm{~km}$ of the plant. Figures 3(a) to 3(d) present predictions made using the median values of the model parameters. The ambient dose equivalent rates (including the background ambient dose equivalent rate) were predicted for the habitation zones of local residents. The forecast suggested that the total area with an annual dose rate of over $20 \mathrm{mSv}(3.8 \mu \mathrm{Sv} / \mathrm{h})$ would decrease within 30 years of the accident to about one-twentieth the level that prevailed five years after the accident. 


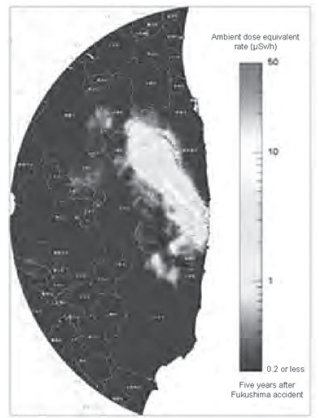

(a) After five years

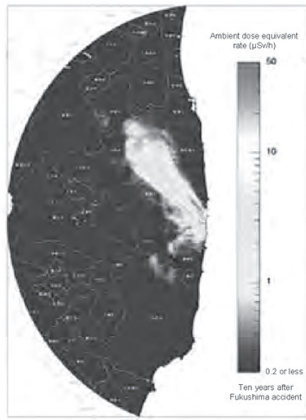

(b) After 10 years

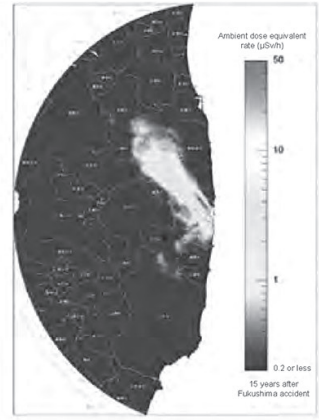

(c) After 15 years

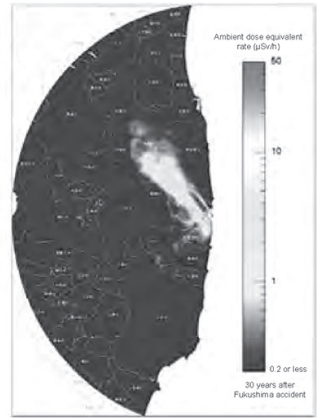

(d) After 30 years

Figure 3 Forecast maps for the distribution of ambient dose equivalent rates

\section{Conclusions}

A model was developed to predict the distribution of ambient dose equivalent rates within $80 \mathrm{~km}$ of the Fukushima Daiichi NPP. A forecast was made using this model for a period of up to 30 years after the nuclear accident. Because the model applies ecological half-lives and other such parameters, it can easily estimate the distribution of ambient dose equivalent rates in habitation zones both inside and outside of the evacuation zones. The model is expected to facilitate the restoration of Fukushima by helping residents to gain an understanding of radiation levels.

This commentary mainly presents the outcomes of the measurement surveys of radioactive materials commissioned by the NRA in FY2014 (consolidation of data on the distribution of radioactive materials produced by the nuclear accident that occurred at TEPCO's Fukushima Daiichi NPP and the development of a migration model). Forecast maps of the ambient dose equivalent rates were generated based on knowledge gained by the JAEA in conducting the commissioned surveys. The distribution prediction model for ambient dose equivalent rates will be revised and reviewed whenever any new measurement data or knowledge is gained.

\section{References}

1) Japan Atomic Energy Agency, Report on the Radiation Measurement Survey Commissioned in FY2012: Study on Distribution and Migration of Radioactive Substances around the Fukushima Daiichi Nuclear Power Plant [in Japanese] (2013), http://fukushima.jaea.go.jp/initiatives/cat03/ entry05.html.

2) Japan Atomic Energy Agency, Report on the Project Commissioned by the Nuclear Regulation Authority in FY2013: Study on Distribution and Migration of Radioactive Substances around the Tokyo Electric Power Company's Fukushima Daiichi Nuclear Power Plant [in Japanese] (2014), http:// fukushima.jaea.go.jp/initiatives/cat03/entry06.html.

3) Japan Atomic Energy Agency, Report on the Project Commissioned by the Nuclear Regulation Authority in FY2014: Consolidation of Distribution Data on the Radioactive Materials Produced by the Nuclear Accident at the Tokyo Electric Power Company's Fukushima Daiichi Nuclear Power Station and Development of a Migration Model [in Japanese] (2015), http://fukushima.jaea.go.jp/initiatives/ cat03/entry07.html. 